\title{
An improved method to study NK-independent mechanisms of MTLn3 breast cancer lung metastasis
}

\author{
Maroesja J. van Nimwegen · Saertje Verkoeijen • \\ Peter J. K. Kuppen · Jurjen H. L. Velthuis · \\ Bob van de Water
}

Received: 28 January 2007/Accepted: 23 April 2007/Published online: 31 May 2007

(C) Springer Science+Business Media B.V. 2007

\begin{abstract}
To study the tumor cell autonomous processes of metastasis, an in vivo tumor metastasis model is required that excludes the involvement of the innate immune system. For this purpose we used the established syngeneic MTLn3 cell - Fischer 344 tumor model. MTLn3 cells are efficiently eradicated by NK cells in vivo. Using isolated cell systems, we provide evidence for apoptosis-induction by IL-2 activated NK cells, but not T-cells, despite the expression of MHC class I. This is largely mediated by the perforin/granzyme B pathway in MTLn3 cells in a caspasedependent manner. Temporal in vivo depletion of NK cells by an antibody-based method, dramatically improved colonization of the lungs by MTLn3 cells, from 5 metastases in the untreated animals to 130 metastases in the NK-depleted animals. Thus, we improved the syngeneic MTLn3Fischer 344 tumor model by temporal depletion of NK cells of which the advantages over the use of immunodeficient animals are evident.
\end{abstract}

Keywords MTLn3 cells - Fischer 344 rats - NK cells · Metastasis

M. J. van Nimwegen - S. Verkoeijen ·

J. H. L. Velthuis - B. van de Water $(\bowtie)$

Division of Toxicology, Leiden Amsterdam Center for Drug Research (LACDR), Leiden University, Leiden, The Netherlands e-mail: b.water@lacdr.leidenuniv.nl

P. J. K. Kuppen

Department of Surgery, Leiden University Medical Center, Leiden, The Netherlands

J. H. L. Velthuis

Department of Immunohematology and Blood Transfusion, Leiden University Medical Center, Leiden, The Netherlands

$\begin{array}{ll}\text { Abbreviations } \\ \text { ADCC } & \text { antibody dependent cellular cytotoxicity } \\ \text { CMA } & \text { concanamycin A } \\ \text { CTL } & \text { cytotoxic T-lymphocyte } \\ \text { E/T } & \text { effector target cell ratio } \\ \text { FACS } & \text { fluorescence activated cell sorter } \\ \text { FAK } & \text { focal adhesion kinase } \\ \text { FRNK } & \text { FAK-related non-kinase } \\ \text { IL-2 } & \text { interleukin 2 } \\ \text { MHC } & \text { Major histocompatibility complex } \\ \text { NK cell } & \text { natural killer cell } \\ \text { PI } & \text { propidium iodide }\end{array}$

\section{Introduction}

In the ongoing search for an anti-cancer drug, numerous in vitro and in vivo models have been generated to study tumor cell biology. Although in in vitro models many aspects of tumor cell biology can be studied, relevant in vivo models are essential to define the ultimate biological relevance of in vitro findings for the intact organism. Thus, the complex environment including local presence of growth factors and chemokines, host immune system and angiogenesis are essential determinants of tumor development and progression including the biological processes essential in metastasis formation [1].

In vivo tumor growth is studied in two different animal models: syngeneic models and immunodeficient models. As in syngeneic models the transplanted cells, the tumor microenvironment, and the host are not only from the same species, but from the same strain of inbred animals, no alloreactivity should occur. Given the importance of tumor 
versus immune cell interactions as principal requirements for tumor progression and metastasis formation, syngeneic models are preferred. However, prolonged culturing of syngeneic tumor cells in vitro may affect the expression of adhesion molecules or antigen expression molecules such as the Major Histocompatibility Complex (MHC) families, rendering these syngeneic cells a target for the immune system. Therefore, other in vivo models, such as the use of immunodeficient mice are being employed. The most used immunodeficient mice (SCID, $\mathrm{Rag}^{-/}$) lack the adaptive immune response, but still harbour large numbers of cells of the innate immune system. Additionally, mice are being used that, due to combined knock out of the Rag-gene and the common gamma chain $\left(\mathrm{Rag}^{-/-} \mathrm{y}_{\mathrm{c}}{ }^{-/-}\right.$), lack both the adaptive and innate immune response.

Two important disadvantages of the use of these immunodeficient animals are the lack of tumor-immune cell interactions and the use of cancer cells originating from other species, i.e. injection of rat cancer cells in mice. In immunocompetent animals, the cytotoxic response of the immune system is mediated by two distinct subsets of lymphocytes: the T-lymphocytes and the Natural Killer (NK) cells. The T-cells belong to the adaptive immune system and respond to foreign antigens presented in self MHC. The NK-cells are an important component of the innate immune system and function in an antigen-nonspecific manner [2]. NK cell-mediated killing of target cells is controlled by a balance between activating and inhibitory signals, of which the latter are mediated by expression of the appropriate MHC I molecules [2]. Both types of lymphocytes kill their targets by excretion of perforin and granzyme B on one hand and by activation of death-receptors on the other hand. All signals finally converge in activation of the executioner caspase-3, leading to induction of apoptosis in the target cells.

The rat mammary adenocarcinoma cell line MTLn3 is an excellent model to mimic breast cancer and its treatment. In the early eighties, Neri and Welch isolated a founder cell line out of the Fischer 344 rat, which was named 13762NF [3]. Injection of this cell line into the fat pad of the Fischer 344 rat resulted in the formation of lung metastases. Several lung metastases were isolated and one of the cultured breasts to lung metastases was named MTLn3. This cell line turned out to be highly metastatic: when injected into the fat pad of female Fischer 344 rats, within 3 weeks the lungs of the rats were burdened with metastases. However, after several passages of in vitro culturing, this cell line started to lose its metastatic potential [4]. To circumvent this problem, immunodeficient mice can be used to study these cells under in vivo conditions, indicating an important role for the immune system in declining metastasis rather than a cell intrinsic phenomenon. Using nude mice, no macro-metastases and only few micrometastases in the lung could be found five weeks after injection of the MTLn3 cells in the fat pad [5], probably due to the NK-mediated killing of metastasizing cells. The importance of NK-cell mediated killing of tumor cells is well established in the MADB106 syngeneic tumor model [6-8] dating back to 1985 [9].

To study the cellular biological processes involved in metastasis, it is important to develop an improved model that excludes interference by the immune system. We have previously used MTLn3 cells to study the mechanisms of cytostatic-induced apoptosis $[10,11]$. In addition, we studied the role of the non-tyrosine kinase focal adhesion kinase in the processes involved in tumorigenesis and metastasis under in vitro circumstances [12]. The goal of the current study was to improve the MTLn3 cell - Fischer 344 rat syngeneic breast tumor metastasis model, to allow the unbiased analysis of tumor cell dependent metastasis programs, independent of the innate immune system surveillance.

A model was set up in which killing of the circulating MTLn3 cells by the innate immune system of Fischer 344 rats was prevented during the metastasis process. In vitro MTLn3 cells are subject to IL-2 activated NK cell-, but not T-cell-mediated killing. Killing depended on granzyme-B/ perforin mediated caspase activation and in vivo depletion of NK cells enabled tail vein injected MTLn3 cells to highly efficient colonize the lungs. In conclusion, by depleting NK cells in Fischer 344 rats, we have created an improved syngeneic model that can be used to study the biological steps taken place in tumor cells that are essential in the formation of distant metastasis.

\section{Materials And Methods}

Animals

Female Fischer 344 rats weighing 70-80 g were purchased from Charles River. They were fed ad libitum and kept at a 12 hour light-dark cycle and kept for at least 2 weeks at the local animal facility before use.

\section{Reagents}

Human recombinant interleukin-2 was obtained from Chiron (Amsterdam, The Netherlands), concanamycin A from Fluka Chemistry (St. Louis, MO, USA) and annexin$\mathrm{V}$ from Roch (Base, Switzerland). Annexin-V was labelled with allophycocyanin (a $652 \mathrm{~nm}$ fluorescent probe) (Prozyme, San Leandro, CA, USA). Molecular Probes (Leiden, The Netherlands) supplied the calcein-AM and the propidium-iodide was purchased from Sigma (St. Louis, MO, USA). Alpha modified minimal essential medium $(\alpha-$ 
MEM), Fetal Bovine Serum (FBS), phosphate buffered saline (PBS) and trypsin were from Life Technologies (Rockville, MD, USA). All other chemicals were of analytical grade.

\section{Cell lines}

MTLn3-tetFRNK cells were used. This cell line is derived from the MTLn3 cell line and differs only from the founder cell line when doxycycline is present; in then expresses a FAK-related non kinase (FRNK) protein [13]. During the experiments described in this paper, no doxycycline is present and therefore, we will refer to this cell line as MTLn3. MTLn3 rat mammary adenocarcinoma cells [3] were cultured as previously described [10].

\section{NK and T-cells}

NK cells were isolated as described previously [14]. Briefly, spleens were aseptically removed from rats and placed in $10 \mathrm{ml}$ culture medium, consisting of RPMI 1640 (L-glutamine, $25 \mathrm{mM}$ HEPES) supplemented with $10 \%$ heat-inactivated FBS, $100 \mu \mathrm{g} / \mathrm{ml}$ streptomycin, $100 \mathrm{IU} / \mathrm{ml}$ penicillin, $5 \mu \mathrm{l}$ lipopolysaccharoids $100 \mu \mathrm{g} / \mathrm{ml}, 6.66 \mathrm{ml} \mathrm{7.5 \%} \mathrm{(w/v)}$ $\mathrm{NAHCO}_{3}, 0.1 \mathrm{ml} 100 \mathrm{mM} \beta$-mercapto-ethanol (complete medium). A single cell suspension was prepared by pressing the spleen through a sterile open filter chamber (Embrarad, Sao Paulo, Brasil). The splenocytes were depleted of Bcells, macrophages and monocytes by allowing these cells to adhere to nylon-wool for 2 hours (Fenwal laboratories, Morton Grove, IL, USA) at $37^{\circ} \mathrm{C}$ in complete medium. The non-adherent cells were collected and cultured in $175 \mathrm{~cm}^{2}$ culture flasks (Greiner, Langentha, Switzerland) in $35 \mathrm{ml}$ culture medium containing 1000 Cetus units human recombinant IL-2 per ml. After 24 hours, the culture medium was transferred to a centrifuge tube and the non-adherent cells were washed three times with $10 \mathrm{ml}$ culture medium without IL-2. The conditioned medium, obtained after centrifugation, was re-added and the cells were cultured in this medium for 6 days. To obtain T-cells, the remaining pellet was resuspended in complete medium containing 100 Cetus units recombinant IL-2 per $\mathrm{ml}$ and cultured in $\mathrm{R} 73$ coated $175 \mathrm{~cm}^{2}$ culture flasks $(6 \mu \mathrm{g} / \mathrm{ml}$ PBS, $6 \mathrm{ml}$ per $\mathrm{TC} 175, \mathrm{o} / \mathrm{n}, 4^{\circ} \mathrm{C}$ ). Two days later, non-adherent cells were removed and complete medium containing 100 Cetus units human recombinant Il-2 per $\mathrm{ml}$ was added. Cells were cultured in this medium for 4 days.

Cytotoxicity assays

MTLn3 cells were harvested and washed twice. They were labelled by incubation with $1 \mu \mathrm{M}$ calcein-AM for
15 minutes in culture medium. For some cytotoxicity assays, MTLn3 cells were pre-incubated with zVAD-fmk $(100 \mu \mathrm{M})$ or DEVD-fmk $(100 \mu \mathrm{M})$ and these inhibitors were also added during the co-incubation period. Subsequently, these cells were washed and seeded in a 96-wellU-bottom plate at a density of 100,000 cells in $0.1 \mathrm{ml} /$ well. The effector cells (NK or T-cells) were added in a volume of $0.05 \mathrm{ml}$. For some experiments, to block induction of apoptosis by the granzyme B/perforin pathway, NK cells were pre-incubated for 2 hours with $20 \mathrm{nM}$ concanamycin A (CMA) which was previously proven to be the optimal concentration to inactivate perforin [14]. After three or six hours, the 96-well plate was centrifuged for 3 minutes at $1500 \mathrm{rpm}$ at $4^{\circ} \mathrm{C}$. The supernatant was removed, the cells were washed with $0.1 \mathrm{ml}$ PBS and the detached cells were transferred to a new plate. Next, $0.1 \mathrm{ml}$ of trypsin was added to the original plate and this plate was incubated for 10 minutes at $37^{\circ} \mathrm{C}$. After trypsinization, the detached cells and the cells in the PBS were pooled and the plate was centrifuged ( $3 \mathrm{~min}$., $1500 \mathrm{rpm} 4^{\circ} \mathrm{C}$ ). Next, the cells were suspended in $200 \mu \mathrm{L}$ binding buffer (10 mM HEPES, $150 \mathrm{mM} \mathrm{NaCl}, 5 \mathrm{mM} \mathrm{KCl}, 1 \mathrm{mM} \mathrm{MgCl} 2.6 \mathrm{H}_{2} \mathrm{O}, 1.8 \mathrm{mM}$ $\mathrm{CaCl}_{2} \cdot 2 \mathrm{H}_{2} \mathrm{O}$ in Milli Q Water, $\left.\mathrm{pH} 7,4\right)$ containing $0.5 \mu \mathrm{l}$ annexin-V $(0.5 \mu \mathrm{g} / \mu \mathrm{l})$. The cells were incubated $(20 \mathrm{~min}-$ utes, $4^{\circ} \mathrm{C}$ ) and subsequently washed twice with binding buffer. Finally, the cells were suspended in $0.2 \mathrm{ml}$ binding buffer and $10 \mu$ propidium-iodide (PI, $100 \mu \mathrm{M}$ ) was added shortly before measurement. Samples were analyzed on the FACScalibur (Becton Dickinson, San Jose, CA). Target cells were selected by gating the calcein-AM positive cells. Viable cells are annexin-V-APC and PI-negative, apoptotic cells are annexin-V-APC positive but PI-negative and necrotic cells are annexin-V-APC- and PI-positive.

\section{MHC-I staining}

MTLn3 cells were harvested and incubated for 30 minutes with $25 \mu \mathrm{l}$ culture supernatant of mAb OX18 IgG (binding to rat MHC class I [15]). The cells were washed with PBS and incubated for 1 hour with the secondary antibody Gtanti mouse-alexa 488 in binding buffer. Finally, the cells were washed and suspended in $0.2 \mathrm{ml}$ binding buffer and $10 \mu \mathrm{l}$ propidium-iodide (PI, $100 \mu \mathrm{M})$ was added shortly before measurement. MHC-I expression was analyzed on the FACScalibur (Becton Dickinson).

In vivo metastasis formation

Cells were injected into the lateral tail vein of Fischer 344 rats as described previously [13]. Briefly, MTLn3 cells were trypsinized, counted, washed twice and resuspended in PBS. $1 \times 10^{5}$ Viable cells in $0.2 \mathrm{ml}$ PBS were injected into the lateral tail vein. After 4 weeks, the animals were 
anesthetized with pentobarbital and the lungs were excised and rinsed in ice-cold PBS. The right lung was cut into three pieces and fixated in isopentane (used to prepare tissue homogenates for immunoblot analysis), 4\% paraformaldehyde or Carnoy's (10\% (v/v) acetic acid, 30\% (v/ v) chloroform in $60 \%$ ethanol). The left lung was injected with ink solution and thereafter destained in water and fixated in Fekete's $(4.3 \%$ (v/v) acetic acid, $0.35 \%$ (v/v) formaldehyde in $70 \%$ ethanol). Lung tumor burden was determined by counting the number of surface metastases.

\section{Purification of mAb3.2.3 antibody}

Culture supernatant was obtained using centrifugation of the hybridoma culture, followed by filtering through a $0.2 \mu \mathrm{M}$ pore size filter. Supernatant was passed through a protein G column (Pharmacia, Uppsala, Sweden). Bound immunoglobulin was eluted using a $\mathrm{pH}$ gradient from $\mathrm{pH}$ 7.4 (PBS) to pH 2.7 (0.1 M Glycin-HCl). Each of the peak fractions obtained was pooled and then dialyzed against PBS. Finally the protein concentration was diluted to $2 \mathrm{mg} /$ $\mathrm{ml}$ in PBS (protein concentration measured against $\mathrm{IgG}$ standard).

\section{Depletion of NK cells}

To deplete NK cells, rats were treated with the monoclonal anti-CD161A antibody, called mAb3.2.3, on 3 consecutive days ( $150 \mu \mathrm{g}$ in $0.5 \mathrm{ml}$ PBS, i.p.). The next day, rats were anesthetized with pentobarbital and at least $1 \mathrm{ml}$ blood was isolated by a heart puncture. After addition of heparin, $2 \mathrm{ml}$ RPMI was added to the blood and this mixture was carefully pipetted on top of $3 \mathrm{ml}$ Ficoll Hypaque and centrifuged (20 min. $2000 \mathrm{rpm}$, room temperature, no brake). The inter phase that contained the white blood cells was isolated and washed 2 times with RPMI. White blood cells were transferred to a V-bottom 96 wells plate, blocked with 5\% BSA in PBS for 30 minutes and incubated with alexa633 labelled mAb3.2.3 in binding buffer. Cells were washed twice with binding buffer and prior to flowcytometric analysis incubated with propidium iodide. Propidium iodide negative (= non-necrotic) cells were gated and using forward-side scatter plots the lymphocytes were gated. By gating the alexa-633 positive cells, the percentage of NK cells was determined in the lymphocyte groups.

\section{Statistical analysis}

Student's t test was used to determine significant differences between two means $(p<0.05)$. For the in vivo lung metastasis experiments, statistical significance was determined by the Mann-Whitney Rank Sum test.

\section{Results}

Intravenous injection of MTLn3 cells does not result in experimental metastasis formation. To study the process of metastasis we used a syngeneic model consisting of the rat mammary adenocarcinoma cell line MTLn3 and the Fischer 344 rats. Initially $1 \times 10^{5}$ MTLn3 cells were injected into the lateral tail vein of the female Fischer 344 rats. However, after 3 weeks, no lung surface tumors were detectable (data not shown). Increasing the number of MTLn3 cells to $5 \times 10^{5}$ and $1 \times 10^{6}$, and prolonging the period to form metastases to 5 weeks did not result in the formation of lung tumors. Only when $1 \times 10^{6}$ cells were injected, a few large lung tumors could be observed 6 weeks post-injection. Importantly, HE stained lung sections did not harbour any dormant tumor cells or micrometastases and those metastases that were present at the surface of the lungs were large. This implicated that even when $1 \times 10^{6}$ tumor cells were injected, few cells survived in the circulation allowing outgrowth into lung metastases. Thus, in our hands, the MTLn3 cell line was not able to colonize the lungs after tail vein injection. Importantly, when these cells were injected subcutaneously in the mammary fat pad, after two weeks, the tumors were palpable and were able to grow out into large tumors in four weeks [13]. Thus, the absence of experimental metastases results from the lack of survival of MTLn3 cells in the circulation.

Syngeneic NK- but not T-cells kill MTLn3 cells

A possible explanation of the lack of lung metastases could be that, despite the use of a syngeneic model, circulating MTLn3 cells are efficiently eradicated by cells of the immune system shortly after injection. As the cytotoxic component of the immune response, responsible for eradication of cellular threats comprises the cytotoxic T-cells and NK-cells, we tested both cell types for their potency to kill MTLn3 cells. Since the tumor cells are very rapidly killed in the circulation, no antigen-specific response could be elicited by the T-cells within that time-frame and therefore, only the possibility of cross-reactivity was tested by examining the cytotoxic potential of polyclonally activated T-cells. To do so, NK cells and T-cells were isolated from the spleen of Fischer 344 rats, activated by culturing in IL-2 enriched medium (and anti-CD3 for T-cells), and followed by an in vitro cytotoxicity assay. For this cytotoxicity assay, the cells were co-incubated for 6 hours at an effector $(=$ NK cells or T-cells)/target $(=$ MTLn3 cells) ratio $(\mathrm{E} / \mathrm{T})$ of 10 . The target cells were labelled with a fluorescent dye, calcein-AM, and prior to the co-incubation, allowing discrimination using FACS analysis (Fig. 1A). After the co-incubation of the target and effector 


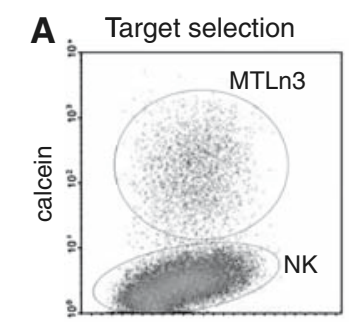

forward scatter

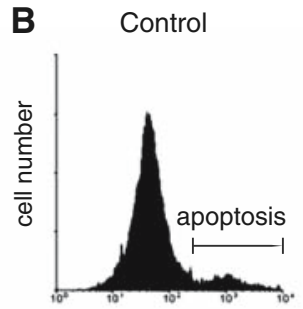

Annexin-633

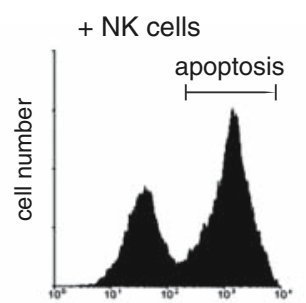

Annexin-633

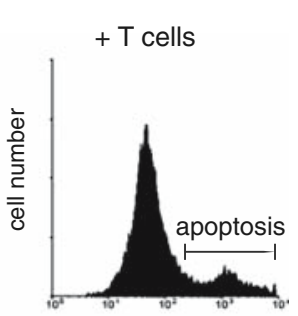

Annexin-633

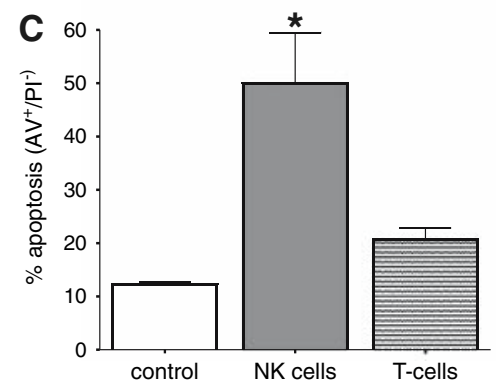

D

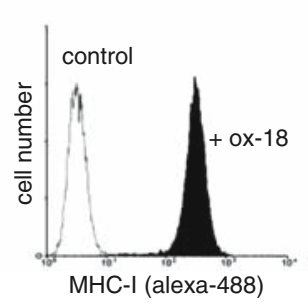

Fig. 1 NK cells, but not T-cells, induce apoptosis in MHC-I expressing MTLn3 cells. MTLn3 cells were labeled with calceinAM and co-incubated with NK or T-cells (E/T 10) for 6 hours, by using flowcytometric analysis, calcein-AM positive MTLn3 cells were gated (A). The flow cytometer was programmed to detect only annexin-633 and PI-staining of calcein-positive MTLn3 cells and the percentage apoptosis (annexin- $633^{+} / \mathrm{PI}^{-}$) was determined in the absence (B, left panel and $\mathbf{C}$ ) and in the presence of NK or T-cells (B, right panels and $\mathbf{C}$ ). MTLn3 cells were incubated with ox-18 and the presence of MHC-I molecules was determined by flow-cytometric analysis (D)

by pre-treatment with CMA, which irreversibly binds perforin. Since co-incubation of the target and effector cells for 6 hours resulted in massive cell death, the coincubation period for this set of experiments was reduced to three hours. Background apoptosis did not exceed $2 \%$ and NK cell induced apoptosis was approximately $19 \%$ (Fig. 2); inhibition of the perforin/granzyme B pathway caused a significant reduction of NK cell-mediated cytotoxicity. To verify that the killing of MTLn3 cells by the

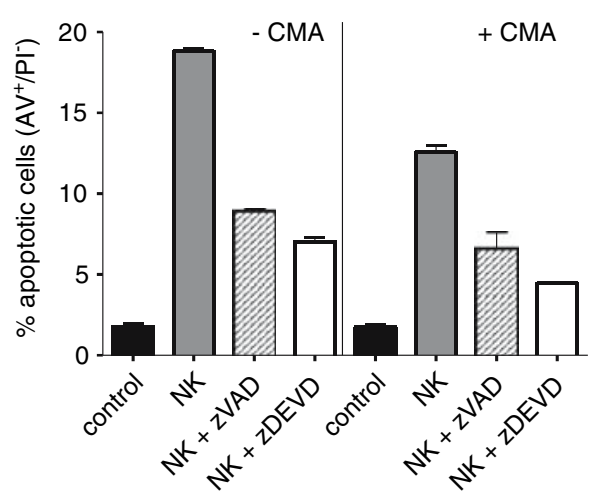

Fig. 2 NK cells induce apoptosis of MTLn3 cells via the granzymeB/perforin pathway. MTLn3 and NK cells were co-incubated as described in figure 1, after 3 hours apoptosis was determined. Where indicated, NK cells were pre-incubated with CMA to prevent granzyme-B/perforin mediated apoptosis and MTLn3 cells were pre-incubated with $100 \mu \mathrm{M} \mathrm{zVAD-fmk} \mathrm{(pan-caspase} \mathrm{inhibitor)} \mathrm{or}$ $100 \mu \mathrm{M}$ zDEVD-fmk (caspase-3 inhibitor) 
NK cells involved the induction of apoptosis, the apoptotic machinery of the MTLn3 cells was abrogated by using a pan-caspase inhibitor (zVAD-fmk) or an inhibitor of the effector caspase-3 (zDEVD-fmk). Previously, we have shown the apoptosis-inhibiting capacity of these caspase inhibitors in cytostatic-induced apoptosis of MTLn3 cells [16]. Pre-treatment of the MTLn3 cells with the caspase inhibitors zDEVD-fmk and zVAD-fmk significantly reduced the percentage of NK cell induced apoptosis. Finally, the combined addition of CMA and caspase inhibition reduced apoptosis almost completely albeit that some residual cell death was present. Again, no differences between experimental and control incubations in propidium iodide positive cells was found. Thus, Fischer 344 NK cells kill MTLn3 cells by induction of apoptosis, predominantly mediated via the granzyme B mediated pathway.

Depletion of NK cells in the Fischer 344 rat enables MTLn3 cells to form metastases

The above data indicate that MTLn3 cells are efficiently killed by NK cells of the Fischer 344 rats by means of apoptosis. Therefore, the lack of lung metastases may well be related to efficient NK cell mediated killing of circulating MTLn3 cells. To confirm the potency of NK cells to prevent metastasis in vivo, NK cells were depleted from the Fischer 344 rats. For this purpose, rats were injected intraperitoneally for three consecutive days with $150 \mu \mathrm{g}$ of the NK depleting antibody, mAb3.2.3 [17]. To determine NK cell depletion, one day after the third intraperitoneally injection blood was isolated from the rats via a heart puncture. Mono-nuclear cells were isolated and stained for the NK-cell specific marker NK1.1, followed by flow cytometric analysis to determine the amount of NK cells. First, using the forward scatter-side scatter plot, the lymphocytes were gated (Fig. 3A, left panel) and subsequently, by gating the NK1.1 positive population, the percentage of NK cells could be determined (Fig. 3A, right panels). The lymphocytes isolated from control animals consisted for approximately $8 \%$ of NK cells (Fig. 3B). Treatment of the rats with the NK depleting antibody reduced the percentage of NK cells to $<0.5 \%$, indicating an efficient depletion of circulating NK cells.

Next, this method was used to determine the role of NK cells in MTLn3 cell killing in vivo, and, consequently, the lack of lung metastasis formation. Therefore, after NK-cell depletion, Fischer 344 rats were injected with only 100,000 MTLn3 cells. All animals were sacrificed after four weeks and the lungs were isolated and injected with ink, as described previously [13]. In control animals, neither lung surface inspection nor microscopical analysis of $\mathrm{H} \& \mathrm{E}$ stained lung sections showed lung tumors and microme-
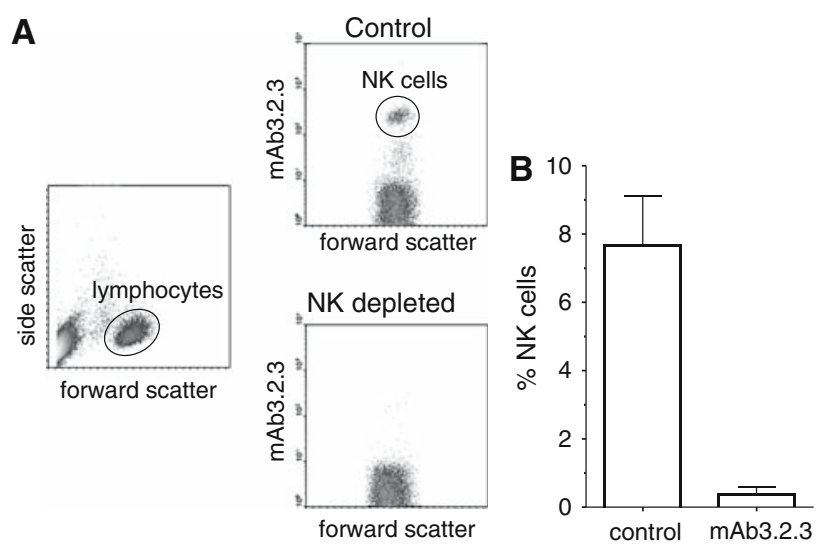

Fig. 3 Pre-treating rats with mAb3.2.3 depletes NK cells from the circulation. Fischer 344 rats $(90-110 \mathrm{~g})$ were intraperitoneally injected with $150 \mathrm{mg}$ mAb3.2.3 on three consecutive days. One day after the last injection, mononuclear cells were isolated from the blood and stained for the NK1.1 specific marker. Using FACS analysis, lymphocytes were gated in a forward scatter-side scatter plot (A, left panel) and the percentage NK cells was determined (A, right panels and $\mathbf{B}$ )

tastases, respectively. Yet, in the lungs of the NK cell depleted animals large numbers of tumors were found (Fig. 4A). In the control group hardly any surface lung metastases were present. However, in the rats that were treated with the NK depleting antibody on average more than 130 lung metastases were present (Fig. 4B and C). In conclusion, temporal depletion of NK-cells from the Fischer 344 rats causes MTLn3 cells to escape immunosurveillance and enables them efficiently to colonize the lungs.

\section{Discussion}

The goal of our study was to improve the MTLn3 cell Fischer 344 rat breast tumor metastasis model, to allow the unbiased analysis of tumor cell dependent metastasis programs in a syngeneic environment. In our hands, MTLn3 cells were not able to form experimental metastases after tail vein injection into syngeneic Fischer 344 rats. We show that MTLn3 cells are efficiently killed by NK cells in a granzyme B and caspase-3 dependent manner. Eradication of NK cells by using the NK cell depleting antibody mAb3.2.3 enables MTLn3 cells to circumvent NK cell killing in the circulation and allows efficient homing of the tumor cells to the lungs followed by outgrowth into metastases. Using this approach, the number of experimental lung surface metastases increased from 5 to 130 upon injection of only $1 \times 10^{5}$ cells, providing an important tool to study the different steps involved in experimental metastasis formation. 


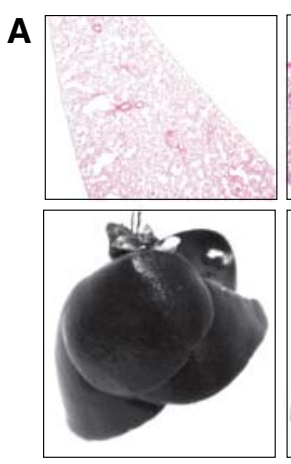

control

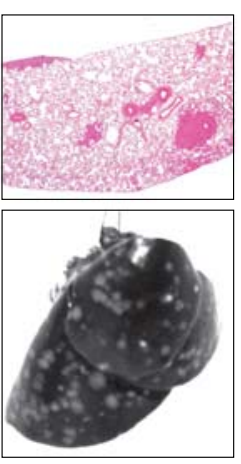

NK depletion

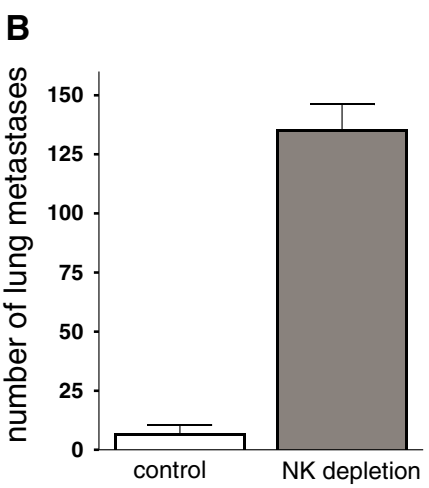

control

\begin{tabular}{|lc|cc|}
\hline Group & Incidence $^{\mathbf{1}}$ & $\begin{array}{c}\text { Number ofmetastases } \\
\text { Median (range) }\end{array}$ & Significance $^{2}$ \\
\hline Control & $3 / 6$ & $2(0-25)$ & \\
NK depletion & $11 / 11$ & $130(57-185)$ & $\mathrm{P}<0.005$ \\
\hline
\end{tabular}

Fig. 4 Depleting NK cell from the circulation enables MTLn3 cells to form experimental metastases. Rats were pre-treated with mAb3.2.3 to deplete NK cells before 100,000 MTLn3 cells were injected into the tail vein. After 4 weeks, lungs were isolated, injected with ink or lung sections were stained with $\mathrm{H} \& \mathrm{E}(\mathbf{A})$ and the number of surface metastases were counted $(\mathbf{B})$. The incidence (number of animals with visible lung surface metastsases/total number of animals in the group), median and significance were determined (C). Statistical analysis was performed by using the Mann-Whitney Rank Sum test

To exclude that the observed lack of metastases after inoculation of $1 \times 10^{6}$ cells was a supplier-specific phenomenon, the experimental metastasis experiments were performed in animals ordered at two different suppliers, Harlan and Charles River. Both experiments did not result in experimental metastases (data not shown). However, when the cells were injected subcutaneously in the mammary fat pad, the cells were able to grow out into large tumors in four weeks [13]. Thus, the lack of experimental metastases could neither be attributed to the used Fischer 344 rats nor to the preparation of the cells. Moreover, the mammary fat pad experiment indicates that the lack of experimental metastases is due to killing of tumor cells in the circulation and not after extravasation into the target organ. Since we think it is very important to study the process of metastasis in a syngeneic model, no experiments in immunodeficient mice were performed. However, injection of MTLn3 cells in the fat pad of nude mice lacking T- but not NK-cells resulted in only low numbers of lung metastases [5]. Thus also in these nude mice, NKcell mediated killing seems to be responsible for the lack of metastases. The importance of Natural Killer cells in the prevention of tumor progression was extensively shown by Ben-Eliyahu and co-workers [6-8]. Using the MADB106 tumor model in Fischer 344 rats they provide evidence for the crucial role for NK-cells in resistance to metastasis and clearly show that external factors influencing susceptibility to malignancies, such as alcohol intoxication, diverse pharmacological agents and stress cause a marked decrease in NK-cell activity $[7,8]$. Concordant with their findings, in 
our experiments, NK-cell mediated killing of circulating tumor cells is responsible for the lack of experimental metastases. Although the eradication of circulating tumor cells could be mediated by NK-or T-cells, our data clearly point toward the NK-cells: First, our in vitro killing assays show that NK-cells, but not polyclonally activated T-cells effectively kill MTLn3 cells. Second, the mammary fat pad experiment [13] shows that intra-organ MTLn3 cells are no longer a target for the immune system. Third, depletion of NK-cells by equivalent injection of 3.2.3 mAb by Van den Brink et al. [17] shows re-appearance of NK- and antibody dependent cellular cytotoxicity (ADCC) activity after 7 days. As the experiments last 28 days, NK cell activity is fully restored one week after tumor cell inoculation. Taken together, these observations show that the MTLn3 cells are a target for the cellular immune system directly upon injection into the circulation, probably lasting no more than 24 hours. Thus, no antigen-specific response of the T-cells towards the MTLn3 cells can be elicited within this limited time-frame. As the T-cells could respond to the MTLn3 cells by cross-reactivity of a memory population, the cytoxocity of polyclonally activated T-cells was tested and disproven. Although these experiments do not formally exclude involvement of T-cell mediated processes in the eradication of MTLn3 cell from the circulation of Fischer 344 rats, our data strongly point towards the Natural Killer cells as the metastases-preventing factor.

NK cell action is controlled by a balance between activating and inhibitory signals, the latter mediated by expression of the appropriate MHC I molecules on the target cells [2]. Flow cytometric analysis showed that MTLn3 cells do express MHC-I molecules. Thus either MTLn3 cells express ligands for the NK cell-activating receptors tipping the balance towards NK reactivity, or the MHC molecules are altered and therefore no longer able to trigger the inhibitory receptors. DNA micro-array data show that MTLn3 cells express RAE-1 (unpublished observations), which is a ligand for the activating receptor NKG2D [19]. The rat homolog of NKG2D, NKR-P2 was shown to bind a RAE-1 like molecule [20]. In conjunction, recently it was shown that NKG2D expression protects the host from tumor initiation [21].

Additionally, a good interaction between target and effector cell is a prerequisite for efficient NK-mediated killing, since blocking antibodies against LFA-1 or ICAM1 strongly inhibited the cytotoxicity of human NK cell clones [22]. Prolonged culturing of the MTLn3 cells may have resulted in the expression of alternative adhesion molecules. Since these surface molecules are important for the target-effector interactions, their changed expression could be responsible for the observed NK cell mediated killing.
Prevention of the granzyme B/perforin mediated apoptotic pathway partly reduced the NK cell-induced cytotoxicity. The remaining apoptosis is probably not mediated via TRAIL or FAS-ligand pathways, since exposure of MTLn3 cells to effector cells expressing either functional TRAIL or FAS-ligand did not result in apoptosis (data not shown); in our hands these effector cells efficiently kill colon tumor cells [23]. The caspase dependency of NK cell-induced cell death in MTLn3-cells was confirmed by addition of zVAD-fmk and DEVD-fmk. Interestingly, prevention of granzyme B-mediated apoptosis in combination with the pan-caspase inhibitor zVAD-fmk, did not completely prevent apoptosis. This might indicate that other proteases, such as cathepsins, may be involved in apoptosis induction by rat NK cells.

Eradication of the NK cells by pre-treating the rats with an NK cell depleting antibody resulted in the formation of more than 130 lung metastases within four weeks after injection of the cells; these results are comparable to in vivo lung metastasis results obtained with earlier passages of the MTLn3 cells [24]. Therefore, the susceptibility of the MTLn3 cells to NK cell cytotoxicity can be regarded as the cause of the lack of lung metastases after tail vein injection of the MTLn3 cells. This model is now used to study cell biological pathways of metastasis. Inducible expression of FRNK, an inhibitor of Focal adhesion kinase (FAK), prevented metastasis formation, providing evidence that this method can indeed be used to study the role of signaling molecules on the process of metastasis [13]. By using inducible expression of the proteins of interest, the importance of proteins in the distinct steps of metastasis can be determined.

In conclusion, we show that NK cells can prevent metastasis in a syngeneic Fischer 344 model, which could be overcome by temporal in vivo depletion of the NK cells, resulting in an improved tumor formation model. The advantages of a syngeneic model over immunodeficient models, such as being more representative for the human situation, are evident.

Acknowledgements The authors thank Chantal Pont, Hans de Bont and Geeske Ensink for their excellent technical assistance. This work was supported by the Dutch Cancer Society, grant 2001/2477 (B.v.d.W.).

\section{References}

1. Khanna C, Hunter K (2005) Modeling metastasis in vivo. Carcinogenesis 26:513-523

2. Lanier LL (1998) NK cell receptors. Annu Rev Immunol 16:35993

3. Neri A, Welch D, Kawaguchi T et al (1982) Development and biologic properties of malignant cell sublines and clones of a 
spontaneously metastasizing rat mammary adenocarcinoma. J Natl Cancer Inst 68:507-517

4. Welch DR, Neri A, Nicolson GL (1983) Comparison of 'spontaneous' and 'experimental' metastasis using rat 13762 mammary adenocarcinoma metastatic cell clones. Invasion Metastasis 3:65-80

5. Bouzahzah B, Albanese C, Ahmed F et al (2001) Rho family GTPases regulate mammary epithelium cell growth and metastasis through distinguishable pathways. Mol Med 7:816-830

6. Melamed R, Rosenne E, Shakhar K et al (2005) Marginating pulmonary-NK activity and resistance to experimental tumor metastasis: suppression by surgery and the prophylactic use of a beta-adrenergic antagonist and a prostaglandin synthesis inhibitor. Brain Behav Immun 19:114-126

7. Ben Eliyahu S, Page GG, Yirmiya R et al (1996) Acute alcohol intoxication suppresses natural killer cell activity and promotes tumor metastasis. Nat Med 2:457-460

8. Ben Eliyahu S, Page GG, Yirmiya R et al (1999) Evidence that stress and surgical interventions promote tumor development by suppressing natural killer cell activity. Int J Cancer 80:880-888

9. Barlozzari T, Leonhardt J, Wiltrout RH et al (1985) Direct evidence for the role of LGL in the inhibition of experimental tumor metastases. J Immunol 134:2783-2789

10. Huigsloot M, Tijdens IB, Mulder GJ et al (2002) Differential regulation of doxorubicin-induced mitochondrial dysfunction and apoptosis by Bcl-2 in mammary adenocarcinoma (MTLn3) cells. J Biol Chem 277:35869-35879

11. Huigsloot M, Tijdens RB, van de Water B (2003) Inhibition of protein kinase Calpha enhances anticancer agent-induced loss of anchorage-independent growth regardless of protection against apoptosis by Bcl-2. Mol Pharmacol 64:965-973

12. van Nimwegen MJ, Huigsloot M, Camier A et al (2006) Focal adhesion kinase (FAK) and protein kinase B cooperate to suppress doxorubicin-induced apoptosis of breast tumor cells. Mol Pharmacol 70:

13. van Nimwegen MJ, Verkoeijen S, van Buren L et al (2005) Requirement for focal adhesion kinase in the early phase of mammary adenocarcinoma lung metastasis formation. Cancer Res 65:4698-4706

14. Velthuis JH, de Bont HJ, Medema JP et al (2003) Interleukin-2 activated NK cells do not use the CD95L- and TRAIL-pathways in the rapid induction of apoptosis of rat colon carcinoma CC531s cells. Immunobiology 207:115-127

15. Fukumoto T, McMaster WR, Williams AF (1982) Mouse monoclonal antibodies against rat major histocompatibility antigens. Two Ia antigens and expression of Ia and class I antigens in rat thymus. Eur J Immunol 12:237-243

16. Huigsloot M, Tijdens IB, Mulder GJ et al (2001) Differential regulation of phosphatidylserine externalization and DNA fragmentation by caspases in anticancer drug-induced apoptosis of rat mammary adenocarcinoma MTLn3 cells. Biochem Pharmacol 62:1087-1097

17. van den Brink MR, Hunt LE, Hiserodt JC (1990) In vivo treatment with monoclonal antibody 3.2.3 selectively eliminates natural killer cells in rats. J Exp Med 171:197-210

18. Velthuis JH, Stitzinger M, Aalbers RI et al (2003) Rat colon carcinoma cells that survived systemic immune surveillance are less sensitive to NK-cell mediated apoptosis. Clin Exp Metastasis 20:713-721

19. Nausch N, Florin L, Hartenstein B et al (2006) Cutting edge: the AP-1 subunit JunB determines NK cell-mediated target cell killing by regulation of the NKG2D-ligand RAE-1epsilon. J Immunol 176:7-11

20. Savithri B, Khar A (2006) A transmembrane-anchored rat RAE1-like transcript as a ligand for NKR-P2, the rat ortholog of human and mouse NKG2D. Eur J Immunol 36:107-117

21. Smyth MJ, Swann J, Cretney E et al (2005) NKG2D function protects the host from tumor initiation. J Exp Med 202:583-588

22. Le Maux CB, Moretta A, Vergnon I et al (2005) NK cells infiltrating a MHC class I-deficient lung adenocarcinoma display impaired cytotoxic activity toward autologous tumor cells associated with altered NK cell-triggering receptors. J Immunol 175:5790-5798

23. Velthuis JH, Rouschop KM, de Bont HJ et al (2002) Distinct intracellular signaling in tumor necrosis factor-related apoptosisinducing ligand- and CD95 ligand-mediated apoptosis. J Biol Chem 277:24,631-24,637

24. Welch DR, Aeed PA, Earhart RH et al (1994) Evidence for paracrine regulation of experimental metastasis in $13762 \mathrm{NF}$ rat mammary adenocarcinoma cell clones. Anticancer Res 14:17431751 